\title{
Matching Breast Lesions in Multiple Mammographic Views
}

\author{
Saskia van Engeland and Nico Karssemeijer \\ University Medical Center St Radboud, Department of Radiology, PO Box 9101, \\ 6500 HB Nijmegen, The Netherlands
}

\section{Introduction}

By combining information from multiple mammographic views (temporal, mediolateral oblique (MLO) and cranio-caudal (CC), or bilateral) it should be possible to improve the accuracy of computer-aided diagnosis (CAD) methods. In literature various approaches have been described to establish correspondence between multiple views. Highnam et al. [1] used a model-based method to find a curve in the MLO view which corresponds to the potential positions of a point in the CC view. Kok-Wiles et al. [2] used a representation of the nested structure of 'salient' bright regions to match mammogram pairs. Karssemeijer et al. [3] and Lau et al. 4] both used a set of landmarks and applied a nonlinear interpolation to align the skin line of two breast images. Almost all matching approaches are based on acquiring a set of landmarks. In a mammogram the nipple is the most obvious landmark. Radiologists use the distance to the nipple to correlate a lesion in MLO and CC view. It is generally believed that this distance remains fairly constant. The goal of this paper is twofold: first, to investigate to what extent this distance remains constant in multiple views, and second, to investigate if the accuracy of automated detection of the nipple is sufficient to use the distance to the nipple as a reliable measure for matching. For this purpose we used an annotated database which contained 327 corresponding mammogram pairs from the Dutch breast cancer screening program.

\section{Method and Preliminary Results}

After segmentation of the mammogram into background and breast tissue, the skin contour can be obtained. Assuming that the location of the nipple is somewhere on this contour, the problem of locating the nipple can be reduced to a one-dimensional problem. When the nipple is clearly visible in profile the curvature of the skin contour will be a good indicator for the nipple position. However, when the nipple is not clearly visible, due to for instance suboptimal positioning or exposure, other features have to be used. Chandrasekhar et al. [5] used an intensity gradient in the direction normal to the skin contour and directed inside the breast for automatically locating the nipple. Previously, at our institute the nipple position was estimated by a relatively simple algorithm that was based on knowledge about the geometry of the breast. After automatically detecting the 
pectoral muscle using the Hough transform, this algorithm determined the point on the skin contour with the largest distance to the pectoral muscle (MLO) or the chest side of the mammogram (CC). Although this approach worked fairly well in 60 percent of the mammograms (error $<6 \mathrm{~mm}$ ), it is not reliable enough to be used for matching lesions in a pair of mammograms.

This paper describes the on-going work on the development of a more accurate method for locating the nipple based on multiple features in combination with a neural network approach. The features that are used describe intensity gradients, shape of the skin contour, line patterns of the glandular tissue, and the geometry of the breast. The feature values are determined for every point on the contour and are fed into a three-layer backpropagation network. The network is trained using a set of 314 mammograms with known nipple position. Figure 1 gives some preliminary results. Although the network still relies very heavily on the distance algorithm, as described above, this new approach has improved the nipple detection accuracy. The variation in the distance to the nipple is approximately the same when using the indicated nipple positions and the nipple positions as determined by the network. To give an indication of the accuracy when using this measure for matching lesions: in 79 percent of the cases the distance to the nipple does not deviate more than $1.5 \mathrm{~cm}$ between both views.
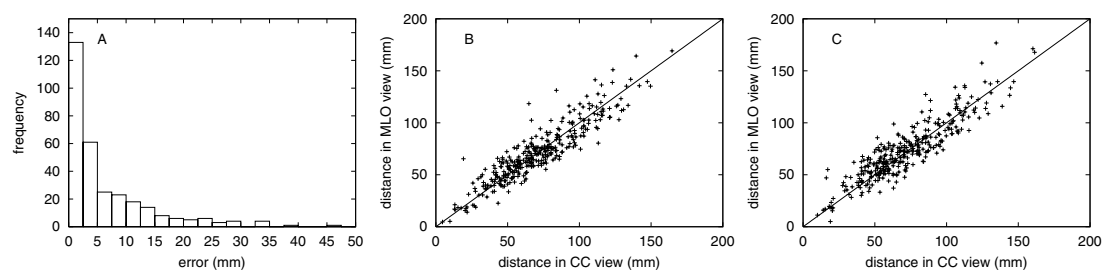

Fig. 1. Histogram of the distance from the actual to the estimated nipple position and scatter plots of the distance $(\mathrm{mm})$ between a lesion and the nipple in $\mathrm{MLO}$ and $\mathrm{CC}$ view using the indicated nipple positions (B) and nipple positions as determined by the network $(\mathrm{C})$.

\section{References}

[1] Highnam, R., Kita, Y., Brady, M., Shepstone, B., and English, R.: Determining correspondence between views. In Digital Mammography, Kluwer, Dordrecht (1998) $111-118$

[2] Kok-Wiles, S., Brady, M., and Highnam, R.: Comparing mammogram pairs for the detection of lesions. In Digital Mammography, Kluwer, Dordrecht (1998) 103-110

[3] Karssemeijer, N., and te Brake, G.: Combining single view features and asymmetry for detection of mass lesions. In Digital Mammography, Kluwer, Dordrecht (1998) 95-102

[4] Lau, Y., and Bischof, W.: Automated detection of breast tumors using the asymmetry approach. Comp and Biomed Research 24 (1991) 273-295

[5] Chandrasekhar, R., and Attikiouzel, Y.: A Simple Method for Automatically Locating the Nipple on Mammograms. IEEE Trans Med Imag 16 (1997) 483-494 